\title{
Experimental Study of Pneumatic Two-Stage Small-Size Radial Turbine
}

\author{
Yuri Pavlovich Kuznetsov ${ }^{1 *}$, Lev Anatolevich Zakharov ${ }^{1}$, Sergey Nikolaevich Khrunkov ${ }^{1}$, Artem Aleksandrovich \\ Kraynov ${ }^{1}$, Aleksandr Evgenevich Zhukov ${ }^{1}$ \\ ${ }^{I}$ Nizhny Novgorod State Technical University n.a. R.E. Alekseev, Minin Str., 24, Nizhny Novgorod, 603950, Russia \\ *Corresponding author E-mail: kuznetsov.y.p@mail.ru
}

\begin{abstract}
This work is aimed at experimental study of the influence of design variables of the first jet reaction stage on the properties of pneumatic two-stage small-size radial turbine. Kinematic layout of the considered turbine is presented, operating processes are described, the final target is formulated to reveal the influence of certain geometrical parameters of the first jet reaction stage which determine overall turbine efficiency. Criterion of nozzle efficiency is determined, variable parameters of multifactorial experiment are selected; experimental facility and procedure of data processing are described. The main experimental results are presented. It is established that the greatest influence on the turbine efficiency is exerted by supersonic nozzle expansion angle. Optimum combination of geometrical expansion extent and geometrical expansion angle of supersonic nozzle of the first jet reaction stage of two-stage small-size radial turbine has been experimentally determined.
\end{abstract}

Keywords: pneumatic drive, turbine drive, jet reaction turbine, Segner wheel, radial turbine, small-size turbine.

\section{Introduction}

Small-size turbines of various kinematic layouts are presently used in engine manufacturing (superchargers) and in cryogenic engineering (helium and hydrogen turboexpanders), in aircraft engineering (cooling turbines, small-size gas turbine engines of drones) and in power engineering (Zysin turbines, small-size turbogenerators), in oil and gas industry (ball valve drive) and in auxiliary mechanisms with pneumatic drive for spacecraft and submersible vehicles, in commercial pneumatic tools.

Numerous designs of small-size turbines have been developed according to various requirements but the most advantageous designs both in terms of manufacturing and efficiency are multistage radial turbines. Blade system of radial pneumatic turbine can be manufactured in press mold by die casting of plastics which significantly reduces its cost upon serial production.

Aiming at manufacture of competitive pneumatic tool, the Chair of power plants and thermal engines, Alekseev State Technical University, Nizhny Novgorod developed and fabricated model series of experimental manual pneumatic grinding machines. Their main feature is the use of innovative two-stage radial turbine where the first stage is comprised of radial jet reaction turbine, and the second one - of radial centripetal turbine [1, 2, 3]. Both stages are embodied on a single disk of operating wheel, which is advantageous in terms of engineering and sizes of the design. This kinematic layout provides efficient aerodynamic performances, velocity and economic characteristics as well as possibility to achieve almost any combination of power and rotation rate. Kinematic layout of small-size two-stage radial turbine is illustrated in Fig. 1.

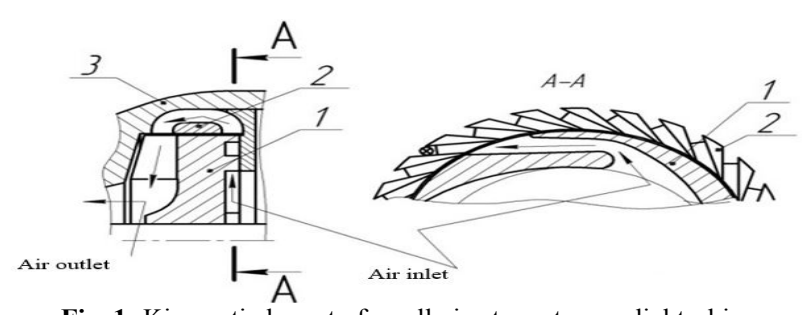

Fig. 1: Kinematic layout of small-size two-stage radial turbine

As illustrated in the figure, working medium (compressed air) is fed to the first stage (position 1) radially from the rotor center. In fixed intermediate guiding apparatus (position 2) the flow gets reversed and reaches the blade system of the second drive stage.

\section{Analysis of Operation Processes}

Predictions of variables of working medium flow along flowing part of the two-stage pneumatic small-size turbine performed in the scope of one-dimension stage theory have demonstrated that the greatest contribution to the overall turbine efficiency is made by the first stage: jet reaction rotating nozzle apparatus [4]. In jet reaction turbine the reaction force is created only by circumferential projection of outlet velocity $[5,6,7]$. With the increase in angle between the outlet velocity vector $C$ and direction of tangent line to operating wheel in the outlet point, the tangent component of reaction force and, hence, turbine efficiency sharply drop. Therefore, in order to increase turbine efficiency in terms of design, it is necessary to provide the minimum angle of flow outlet. Triangles of velocity vectors at skewed nozzle outlet are illustrated in Fig. 2. 


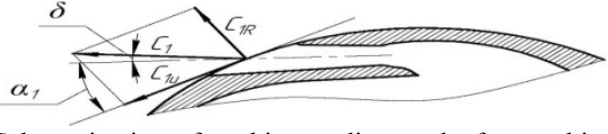

Fig. 2: Schematic view of working medium outlet from turbine nozzle

Contradictory impact of various design nozzle parameters of the first jet reaction stage (such as the ratio of inlet to outlet nozzle surface areas, nozzle length, position of nozzle axial line and some others) on the properties of pneumatic small-size radial turbine requires for experimental studies. The necessity to study efficiency of jet reaction turbine is also related with the dimensions: in small-size turbines, due to high relative roughness and relative gaps, operation processes can be described insufficiently using conventional predictions of pneumatic turbines.

\section{Design of Experiment}

Velocity factor of nozzle apparatus of jet reaction turbine (the ratio of actual flow rate of working medium from nozzle to isentropic flow rate from the same nozzle) was selected as the target function. In order to reduce expenses for experiments, the experimental design theory was applied. Variables (the considered factors) of jet reaction turbine were selected on the basis of analysis of a priori information on the influence of factors. Among all geometric factors affecting turbine efficiency, such as geometric angle of flow exit, expansion angle of supersonic nozzle, length of nozzle sub- and supersonic portions, decrease rate of air pressure in supersonic portion, the most significant ones were selected. It is known that the variables of supersonic nozzle have more significant influence on the efficiency of its operation than the variables of subsonic portion. Hence, two factors were selected for the experiments: geometrical expansion angle of supersonic portion $\gamma$ and geometrical channel expansion rate in supersonic portion $F / f$, which was the ratio of outlet surface area of the nozzle 1 to its critical surface area 2, Fig. 3. Other assumed factors had either lower or known influence on the target function, hence, they were assumed constant in the experiments.

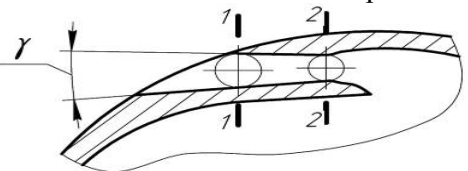

Fig. 3: Factors for studying nozzle efficiency

The next task was determination of domain of the selected factors. Expansion angle of supersonic portion can vary from $\gamma=0^{\circ}$ to $\gamma=$ $40^{\circ}$. These limits are of engineering character, since it is required to install several nozzles on constrained surface of operating wheel, leaving free segments in order to vary this factor. From the same considerations, the domain boundaries of geometrical channel expansion rate in supersonic portion $F / f$ are from 1.0 to 1.6 .

The design of the first series of two-factor experiment, according to the steepest ascent method, included 5 combinations of factors. They were comprised of one reference point (point $« 0 »)$ and 4 points in the vicinities, covering all 4 main possible directions of gradient vector. In order to define correctly parameters of reference point, a priori information was very important. From the point of view of gas dynamics, the optimum values of the selected factors for reference point were as follows: $\gamma=8 \ldots 12^{\circ}, F / f=1,53[8$, $9,10,11,12,13,14]$, Fig. 4. In the first experimental series the step for geometrical expansion rate $F / f$ was set to $\Delta_{\mathrm{F} / \mathrm{f}}=0.1$, and the step of geometrical expansion angle of supersonic portion $\gamma$ was set to $\Delta_{\gamma}=2^{\circ}$. According to the experimental design, 5 operating wheels were developed and manufactured for the first experimental series. The wheels were manufactured by $3 \mathrm{D}$ printing. Drawings and photos of the experimental operating wheels are shown in Fig. 5.

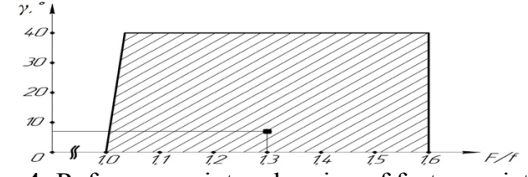

Fig. 4: Reference point and region of factor variation

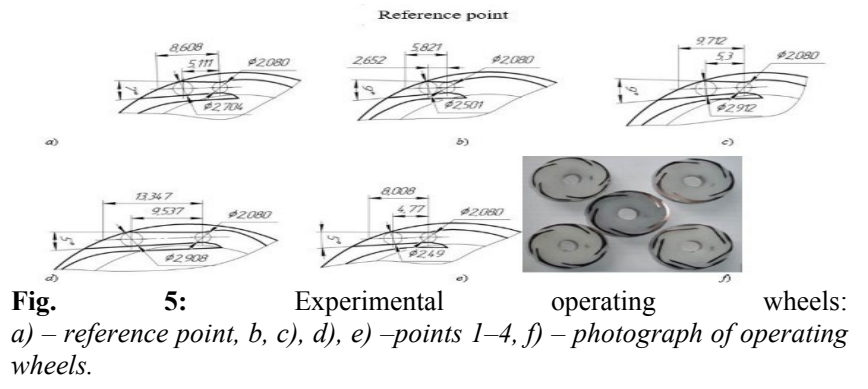

\section{Experimental Facility}

The main properties of experimental turbines were determined on the experimental facility for stages and blade systems of smallsize turbines [15] illustrated in Fig. 6. The considered nozzle apparatus 5 is mounted in the body 4 into the coupling of gas static suspension 8 which, due to gas layer, prevents mechanical contact with the fixed rim 7 thus improving the accuracy of measurements. Air is supplied to the gas static suspension via the tubes 2 . The working medium is supplied to the considered nozzle apparatus via 3 tubes, position 3, located radially to the receiver in $120^{\circ}$ in the plane perpendicular to cylinder axis. The labyrinth sealings 6 are intended for decrease in air leakage from air supply system to the nozzle apparatus. The torque created by the nozzle apparatus is transferred to the moving coupling of the gas static suspension 8 and is detected by the torque strain sensor 10 .

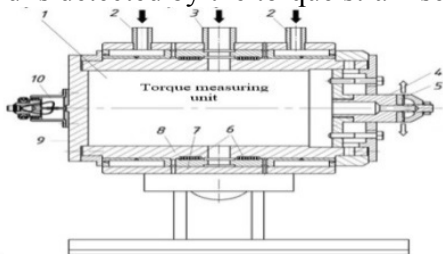

Fig. 6: Experimental facility for studying the nozzle apparatuses: 1 receiver; 2 - air inlet tube to gas static suspension; 3 - air inlet tube to the considered nozzle apparatus; 4 - body for mounting of nozzle apparatus; 5 - the considered nozzle apparatus; 6 - labyrinth sealing; 7 - static rim of gas static suspension; 8 - coupling of gas static suspension; 9 - flange of gas static suspension; 10 - torque strain sensor.

While measuring torque created by nozzle apparatus, the torque sensor of the facility detects cumulative flow rate of working medium (flow rate across the nozzle apparatus and leakages through labyrinth sealing) at pressure $P_{0}$. In order to prevent leakage through labyrinth sealing during measurements of flow rate across the nozzle apparatus, a special device is used for direct supply of working medium to the nozzle apparatus bypassing sealing of gas static suspension and the torque measuring unit while retaining pressure before the nozzle apparatus.

The layout of supply system and measuring instruments of the experimental facility are illustrated in Fig. 7, overall view of the facility - in Fig. 8. The manual tap, the air filter 2, and the manometer 3 are installed in inlet channel of the supply system. The supply system is subdivided into 2 lines separated from the inlet channel by the electropneumatic valves 4 and 5 which protect the facility against accidental starts with deactivated control system. The first main line is intended for air supply to gas static suspension, and the second one - to the experimental facility. The second pipeline is equipped with the electropneumatic converter 6 , which controls the experiments, and with the instruments to measure air flow rate and temperature. Therefore, the facility can 
measure toque created by the nozzle apparatus, as well as pressure, temperature, and flow rate of working medium.

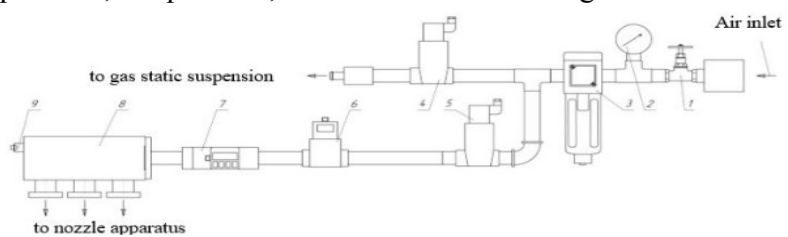

Fig. 7: Schematic view of supply system and measuring instruments of the facility:

1 - manual tap; 2 -K4-10-50 manometer; 3 -EAF6000 filter; 4,5 pneumatic valve; $6-$ ITV3050-21F4N electropneumatic converter with pressure indication; 7 -PF2A703H-F10-28N flow meter; 8 - air inlet collector to torque measuring unit; $9-\mathrm{DS} 18 \mathrm{~B} 20$ temperature sensor.

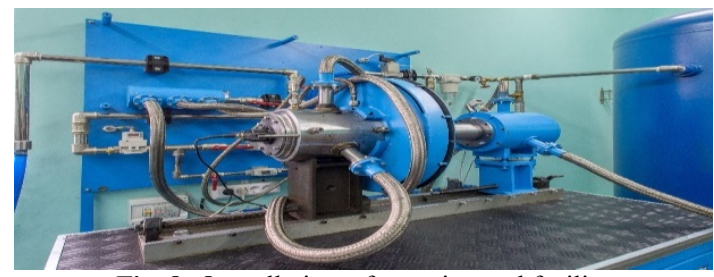

Fig. 8: Overall view of experimental facility

Integral parameters of the nozzle apparatus were predicted as follows. The circumferential component of absolute velocity at outlet from the nozzle apparatus $C_{1 u}$ is determined by equation of flow torque in the nozzle apparatus:

$C_{1 u}=\frac{M}{R_{e x t} G}$,

where $M$ is the torque measured on the experimental facility; $R_{\text {ext }}$ is the external radius of the centrifugal nozzle apparatus (in the experiment: $\mathrm{R}_{\mathrm{ext}}=44 \mathrm{~mm}$ ); $G$ is the gas mass flow rate.

The radial component of absolute velocity $C_{1 r}$ at outlet from the nozzle apparatus is determined by the continuity equation:

$C_{1 r}=\frac{G}{F \rho_{1}}$,

where $G$ is the mass flow rate; $F$ is the surface area of nozzles determined by outlet cross sections of nozzles; $\rho_{1}$ is the real gas density in outlet cross sections of nozzles.

Since the gas density $\rho_{1}$ at outlet from the nozzle apparatus in actual process is unknown, then the additional interrelations between the flow variables should be used for determination of $C_{1 r}$.

Let us consider the following method of determination of integral parameters. Since the initial parameters of isentropic and actual processes coincide, then the loss in nozzles can be determined as follows:

$\zeta=i_{1}-i_{i s}=C_{p}\left(T_{1}-T_{i s}\right)=C_{p}\left(1-\psi^{2}\right)\left(T_{0}^{*}-T_{1 i s}\right)$,

hence:

$$
T_{1}=T_{1 i s}\left[1+\left(1-\psi^{2}\right)\left(\frac{T_{0}^{*}}{T_{1 i s}}-1\right)\right],
$$

where $\zeta$ is the energy loss during actual expansion in the nozzle apparatus; $i_{1}$ is the enthalpy in final cross section of the nozzle apparatus upon actual expansion $; i_{i s}$ is the enthalpy in final cross section of the nozzle apparatus upon isentropic expansion; $C_{p}$ is the air mass isobar heat capacity; $T_{1}$ is the temperature in the end of actual air expansion in nozzles; $T_{i s}$ is the temperature in the end of isentropic air expansion in nozzles; $\psi$ is the velocity factor.

The temperature in the end of isentropic air expansion in nozzles is determined by the equation of adiabatic expansion:

$T_{1 i s}=T_{0}^{*}\left(\frac{P_{a}}{P_{0}^{*}}\right)^{\frac{k-1}{k}}$

where $T_{0}{ }^{*}$ is the air braking temperature before the nozzle apparatus; $p_{a}$ is the air ambient pressure; $p_{0}{ }^{*}$ is the initial total air pressure before the nozzle apparatus; $k$ is the adiabatic exponent.

From the equation of state for gas parameters in the nozzle outlet cross section, let us determine the gas density at outlet from the nozzle apparatus:

$\rho_{1}=\frac{P_{a t m}}{R T_{1}}$

where $R$ is the gas constant, for air: $\mathrm{R}=287 \mathrm{~J} / \mathrm{kg} \cdot \mathrm{K}$.

Then, with consideration for Eqs. (3), (4), and (5), Eq. (2) is rewritten as follows:

$C_{1 r}=\frac{G R}{F P_{a t m}} T_{0}^{*}\left(\frac{P_{a}}{P_{0}^{*}}\right)^{\frac{k-1}{k}}\left[1+(1-\psi)\left(\frac{T_{0}^{*}}{T_{1 i s}}-1\right)\right]$.

By definition the nozzle velocity factor is:

$\psi=\frac{\sqrt{C_{1 u}^{2}+C_{1 r}^{2}}}{C_{1 i s}^{2}}$

Finally, we have a set of 2 equations, Eqs. (6) and (7), with 2 unknown variables: $\psi$ and $C_{1 r}$. The set is solved by iterations. Knowing the velocity projections, it is possible to predict the angle between the vector of flow outlet velocity and the tangential line to operating wheel at the flow outlet point.

\section{Results}

Taking into consideration the design requirements in the first experimental series, the following combinations of geometric variables of operating wheel were used, see Table 1. Table 2 summarizes the main results of the first experimental series. The surface area of flow cross section of the nozzle apparatus for various operating wheels was from 13.98 to $15.41 \mathrm{~mm}^{2}$, the air temperature: $+23^{\circ} \mathrm{C}$, the ambient pressure: $0.100 \mathrm{MPa}$, the pressure before the nozzle apparatus: $0.321 \mathrm{MPa}$.

Table 1: $F / f$ and $\gamma$ factors for the first experimental series

\begin{tabular}{|c|c|c|}
\hline Point No. & Adopted absolute $F / f$ & Adopted absolute $\gamma$ \\
\hline 0 & 1.3 & 7 \\
\hline 1 & 1.2 & 9 \\
\hline 2 & 1.4 & 9 \\
\hline 3 & 1.4 & 5 \\
\hline 4 & 1.2 & 5 \\
\hline
\end{tabular}

Table 2: Main results of the first experimental series

\begin{tabular}{|c|c|c|c|c|c|}
\hline No. & $\begin{array}{c}\text { Air flow } \\
\text { rate through } \\
\text { nozzle } \\
\text { apparatus } \\
(\mathrm{G}, 1 / \mathrm{min})\end{array}$ & $\begin{array}{c}\text { Nozzle } \\
\text { apparatus } \\
\text { torque } \\
\left(\mathrm{M}_{0}, \mathrm{~N} \cdot \mathrm{m}\right)\end{array}$ & $\begin{array}{c}\text { Velocity } \\
\text { factor of } \\
\text { nozzle } \\
\text { apparatus } \\
(\psi)\end{array}$ & $\begin{array}{c}\text { Flow } \\
\text { exit } \\
\text { angle } \\
\left(\gamma,{ }^{\circ}\right)\end{array}$ & $\begin{array}{c}\text { Flow } \\
\text { coefficient } \\
\text { of nozzle } \\
\text { apparatus } \\
(\mu)\end{array}$ \\
\hline 0 & 740 & 0.095 & 0.785 & 33.286 & 0.968 \\
\hline 1 & 705 & 0.098 & 0.825 & 30.586 & 0.973 \\
\hline 2 & 715 & 0.090 & 0.748 & 30.842 & 0.974 \\
\hline 3 & 675 & 0.085 & 0.749 & 31.094 & 0.977 \\
\hline 4 & 715 & 0.089 & 0.787 & 36.600 & 0.974 \\
\hline
\end{tabular}

On the basis of the obtained results, the regression equation of the first order was derived. The linear response function was adopted in the first experimental series: $\psi=a \cdot(\gamma)+b \cdot(F / f)+c$, 
since such form was required and sufficient for determination of the function gradient. The obtained regression equation was as follows:

$$
\psi=0.01075 \cdot(\gamma)-0.03075 \cdot(F / f)+0.7818
$$

The gradient of response function based on the results of the first experimental series is illustrated in Fig. 8. In the considered domain of parameters it is possible to conclude that the velocity factor depends more on the geometrical expansion extent than on the geometrical expansion angle of supersonic nozzle of jet reaction turbine.

For subsequent specification of optimum geometrical variables of the nozzle apparatus, it is required to consider the combinations of variable factors in accordance with direction of the aforementioned gradient of the response function, see Fig. 9. With consideration for design requirements in the second experimental series, the combinations of geometrical variables of operating wheels in Table 3 were adopted.

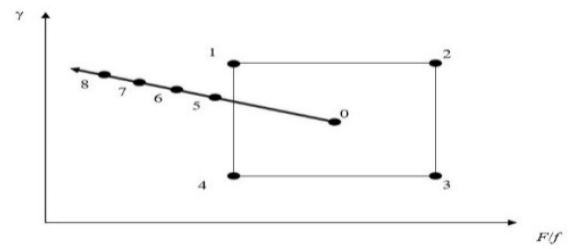

Fig. 9: Gradient of factors and experimental points

All experimental lines were positioned on the gradient line, each subsequent point at higher distance from the reference point " 0 ", Fig. 9. Surface area of the flow cross section of the nozzle appratus for various operating wheels was in the range from 14.56 to $15.01 \mathrm{~mm}^{2}$, the air temperature: $+20^{\circ} \mathrm{C}$, the atmospheric pressure: $0.101 \mathrm{MPa}$, the pressure between the nozzle apparatus: $0.320 \mathrm{MPa}$. The main experimental results of the second series are summarized in Table 4.

Table 3: $F / f$ and $\gamma$ for the second experimental series

\begin{tabular}{|c|c|c|}
\hline Point No. & Adopted absolute $F / f$ & Adopted absolute $\gamma$ \\
\hline 5 & 1.27 & 10 \\
\hline 6 & 1.26 & 12 \\
\hline 7 & 1.25 & 14 \\
\hline 8 & 1.23 & 16 \\
\hline
\end{tabular}

Table 4: Main results of the second experimental series

\begin{tabular}{|c|c|c|c|c|c|}
\hline No. & $\begin{array}{c}\text { Air flow rate } \\
\text { through } \\
\text { nozzle } \\
\text { apparatus }(\mathrm{G}, \\
1 / \mathrm{min})\end{array}$ & $\begin{array}{c}\text { Nozzle } \\
\text { apparatus } \\
\text { torque }\left(\mathrm{M}_{0}\right. \\
\mathrm{N} \cdot \mathrm{m})\end{array}$ & $\begin{array}{c}\text { Velocity } \\
\text { factor of } \\
\text { nozzle } \\
\text { apparatus } \\
(\psi)\end{array}$ & $\begin{array}{c}\text { Flow } \\
\text { exit } \\
\text { angle } \\
\left(\gamma^{\circ}\right)^{\circ}\end{array}$ & $\begin{array}{c}\text { Flow } \\
\text { coefficient of } \\
\text { nozzle } \\
\text { apparatus }(\mu)\end{array}$ \\
\hline 5 & 715 & 0.097 & 0.798 & 30.534 & 0.968 \\
\hline 6 & 695 & 0.102 & 0.832 & 26.550 & 0.970 \\
\hline 7 & 725 & 0.096 & 0.779 & 30.518 & 0.972 \\
\hline 8 & 710 & 0.093 & 0.766 & 29.868 & 0.975 \\
\hline
\end{tabular}

Velocity factor of the nozzle apparatus increased up to point 6 and then decreased. Therefore, while moving along the gradient direction, the maximum of target function was passed and the optimum combination of the considered geometric variables of nozzle apparatus was determined.

\section{Conclusion}

While analyzing experimental data, it should be mentioned that the greatest influence on the nozzle efficiency is exerted by geometrical expansion angle of supersonic nozzle of jet reaction turbine $\gamma$, this is indicated by the coefficients of appropriate variables in regression equation. The obtained results agree with the data of other researchers $[16,17,18,19,20,21]$ and provide additional information. Herewith, the experimental point 6 provides the greatest efficiency of pneumatic two-stage small-size radial turbine. Optimum combination of nozzle variables of the first jet reaction stage of the considered turbine was determined experimentally:

$\gamma=12^{\circ}, F / f=1.26$

\section{References}

[1] Y.P. Kuznetsov, V.L. Khimich, S.N. Khrunkov, et al., Radial two-stage microturbine for pneumatic actuation, Russian Aeronautics 59(2) (2016) 283-286.

[2] V.L. Khimich, A.B. Chuvakov, V.A. Kikeyev, et al., Two-rimming radial turbine for drive of manual pneumatic grinders, International Journal of Applied Engineering Research 11(16) (2016) 8982-8986.

[3] V.L. Khimich, A.B. Chuvakov, S.N. Khrunkov, Maximum rotation frequency regulators of high-velocity small-sized pneumatic actuators, International Journal of Applied Engineering Research 11(18) (2016) 9256-9260.

[4] V.L. Khimich, A.B. Chuvakov, S.N. Khrunkov, et al., The influence of aerodynamic characteristics of the elements of the flow range of the radial two-row range of the radial two-row microturbine on its dynamic characteristics, International Journal of Applied Engineering Research 11(23) (2016) 11501.

[5] I.I. Kirillov, A.I. Kirillov, Teoriva turbomashin [Theory of turbomachines], Mashinostroenie, Leningrad, 1974.

[6] A.S. Natalevich, Vozdushnye mikroturbiny [Air microturbines], Mashinostroenie, Moscow, 1979.

[7] M.E. Deich, Tekhnicheskaya gazodinamika [Engineering gas dynamics], Energiya, Moscow, 1974.

[8] A.Yu. Fershalov, M.Yu. Fershalov, Yu.Ya. Fershalov, et al., Results of the study rotor wheels supersonic microturbines with a large angle of rotation of the flow, Applied Mechanics and Materials 752-753 (2015) 884-889.

[9] Yu.Ya. Fershalov, M.Yu. Fershalov, A.Yu. Fershalov, Energy efficiency of nozzles for axial microturbines, Procedia Engineering 206 (2017) 499-504

[10] A.Yu. Fershalov, M.Yu. Fershalov, Yu.Ya. Fershalov, et al., Research data of turbine nozzles of 5-9 degree outlet angles, Applied Mechanics and Materials 770 (2015) 547-550.

[11] A.Yu. Fershalov, M.Yu. Fershalov, Yu.Ya. Fershalov, et al., The design of the nozzle for the nozzle box microturbines, Applied Mechanics and Materials 789-790 (2015) 203-206

[12] Yu.Ya. Fershalov, T.V. Sazonov Experimental research of the nozzles, Advanced Materials Research 915-916 (2014) 345-348.

[13] T.V. Sazonov, Y.Y. Fershalov, M.Y. Fershalov, et al., Experimental installation for the study of nozzles microturbines, Applied Mechanics and Materials 635-637 (2014) 155-158.

[14] D. Ibragimov, A. Mochalov, Yu. Ilinskiy, Research Data of Microturbine Nozzles with Outlet Angles under 9 Degree (Conference Paper), International Conference on Industrial Engineering, ICIE 2017; Saint-Petersburg; Russian Federation; 16 May 2017 to 19 May 2017; Procedia Engineering 206 (2017) 493-498

[15] Yu.P. Kuznetsov, A.B. Chuvakov, Eksperimental'naya ustanovka dlya issledovaniya malorazmernykh turbinnykh stupenei [Experimental facility for investigation into small-size turbine stages], Izvestiya VUZov. Mashinostroenie, 4 (2013) 58-64.

[16] V.I. Korenbaum, A.A. Tagiltsev, A.E. Kostiv, et al., A low-frequency power-type pressure-gradient receiver for oceanological investigations, Instruments and Experimental Techniques 60 (2017) 5.

[17] Fershalov, Yu. Fershalov, M. Fershalov, et al., Constructive and regime factors influence on turbine wheel characteristics with large rotation flow angle of blades, Polyarnaya mekhanika 3 (2016) 976-985.

[18] A.Yu. Fershalov, Yu.Ya. Fershalov, L.P. Tsigankova, The degree of influence of constructive and regime factors on the characteristics turbine wheel steps shoulder who are more angles of rotation, RECENT ADVANCES in MATHEMATICS, Series "Mathematics and Computers in Science and Engineering Series", WSEAS Press, Budapest, 2015, 130-133.

[19] A.Y. Fershalov, Y.Y. Fershalov, M.Y. Fershalov, et al., Analysis and optimization of efficiency rotor wheels microturbines, Applied Mechanics and Materials 635-637 (2014) 76-79.

[20] M.Y. Fershalov, Y.Y. Fershalov, A.Y. Fershalov, et al., Microturbines degree of reactivity, Applied Mechanics and Materials 635-637 (2014) 354-357.

[21] Yu.Ya. Fershalov, Technique for physical simulation of gasodynamic processes in the turbomachine flow passages, Russian Aeronautics 55(4) (2012) 424-429. 